\title{
VARIABILIDAD ESPACIO-TEMPORAL DE LAS SEQUÍAS EN LA CUENCA PACÍFICO NORTE DE MÉXICO (1961-2010)
}

\author{
L. SERRANO-BARRIOS ${ }^{1 *}$, S.M. VICENTE-SERRANO ${ }^{2}$, \\ H. FLORES-MAGDALENO ${ }^{1}$, L. TIJERINA-CHÁVEZ ${ }^{1}$, D. VÁZQUEZ-SOTO ${ }^{1}$ \\ ${ }^{1}$ Colegio de Postgraduados, Montecillo, México. \\ ${ }^{2}$ Instituto Pirenaico de Ecología, Consejo Superior de Investigaciones \\ Científicas (IPE-CSIC), Zaragoza, España.
}

\begin{abstract}
RESUMEN. En este trabajo se analiza la variabilidad espacio-temporal de las sequías en la Cuenca Pacífico Norte de México entre 1961 y 2010, mediante dos indices de sequía: el Índice de Precipitación Estandarizada (SPI) y el Índice de Precipitación Evapotranspiración Estandarizada (SPEI). Para ello se han utilizado 48 estaciones meteorológicas con datos de precipitación, temperatura máxima y mínima mensual, de ellas en 22 estaciones también se dispuso de datos de evaporación media medida mediante evaporímetros Piché. La evapotranspiración de referencia, necesaria para calcular el SPEI, se obtuvo mediante la ecuación de Hargreaves. Se ha comprobado que la sequía es un fenómeno frecuente en la Cuenca del Pacífico Norte y que independientemente del impacto del posible estrés relacionado con la evapotranspiración, se registran episodios numerosos, algunos de ellos de gran intensidad, asociados a anomalías de precipitación durante largos periodos. Los resultados muestran que los principales episodios de sequía ocurrieron en las décadas de 1980 y 2000, aunque existe una notable variabilidad espacial a lo largo de la región. Se discute el posible impacto de la demanda de agua por parte de la atmósfera en la evolución de la sequía observada con dichos índices, y sus implicaciones para una adecuada valoración del riesgo.
\end{abstract}

Spatio-temporal variability of droughts in the North Pacific Basin of México (1961-2010)

ABSTRACT. This article analyses the spatio-temporal variability of droughts in the North Pacific Basin of México between 1961 and 2010, using two drought indices: the Standardized Precipitation Index (SPI) and the Standardized Precipitation Evapotranspiration Index (SPEI). We used data from 48 weather stations with available data of precipitation and monthly minimum and maximum temperature. In 22 of the weather stations, time series of Piché evaporation were also available. The reference evapotranspiration, necessary to obtain the SPEI, 
was calculated by means of the Hargreaves equation. Results show that major droughts occurred in the 1980s and 2000s, although there is a noticeable spatial variability across the region. Finally, the potential impact of the atmospheric evaporative demand on drought severity observed by the different drought indices is discussed, and the possible implications for an appropriate risk assessment.

Palabras clave: sequía, Índice de Precipitación Estandarizada, Índice de Precipitación Evapotranspiración Estandarizada, evolución temporal, diferencias espaciales, tendencias.

Key words: drought, Standardized Precipitation Index, Standardized Precipitation Evapotranspiration Index, temporal evolution, spatial differences, trends.

Recibido el 21 de septiembre de 2015 Aceptado el 22 de diciembre de 2015

*Correspondencia: Liliana Serrano-Barrios, Colegio de Postgraduados, Carretera México-Texcoco, Km 36.5, Montecillo, México. E-mail: liliana18458@gmail.com

\section{Introducción}

La sequía es un fenómeno natural muy complejo debido a sus características (Wilhite, 2000), con problemas de definición y cuantificación muy importantes (Wilhite y Glantz, 1985; Vicente-Serrano et al., 2011; Lloyd-Hughes, 2014), aspecto que dificulta enormemente su estudio. Una sequía está compuesta por tres componentes fundamentales: magnitud (déficit acumulado por debajo de un determinado umbral), duración (número de meses o años consecutivos por debajo de un determinado umbral) e intensidad (déficit medio del periodo que está por debajo del umbral) (Dracup et al., 1980). Mediante la utilización de índices de sequía climáticos (Mishra y Singh, 2010), es posible tener una idea de la severidad de las sequías y cuantificar dichos componentes, su variabilidad en el tiempo y sus tendencias temporales.

En México, la sequía es un riesgo climático de primer orden, con importantes implicaciones ecológicas, económicas y sociales (Mullholand et al., 1997; Laliberte et al., 2004). Las sequías han sido un hecho recurrente en todo el territorio mexicano, existiendo fundadas evidencias de que la elevada recurrencia del fenómeno puede haber afectado el desarrollo histórico de las civilizaciones en la región (Curtis et al., 1996; Hodell et al., 2011). Durante el siglo XX e inicios del siglo XXI se han registrado sequías muy severas en México (Magaña et al., 1999; Mendoza et al, 2006; Méndez y Magaña, 2010; Bhattacharya y Chiang, 2014), fundamentalmente en las décadas de 1930 (extensibles también a amplias zonas de Norteamérica), 1950, 1990 y 2000 (Trenberth y Guillemot, 1996). En la región del Organismo de Cuenca Pacífico Norte la presencia de sequías recurrentes en las últimas décadas ha provocado importantes impactos negativos en los sectores agrícola, ambiental y en todas las actividades socio-económicas en general 
(Ravelo et al., 2014). Además, las recientes sequías de gran severidad han aumentado el riesgo de sobreexplotación acuífera permanente y el fenómeno de la intrusión salina, generando tensiones sociales por el agua disponible, manifestadas por robos de agua en canales y en una gran cantidad de perforaciones de pozos clandestinos (Douriet, 2013).

Existen estudios que sugieren que el proceso de calentamiento observado en el área de estudio puede estar reduciendo notablemente la disponibilidad de recursos hídricos en la región. De hecho, Seager y Vecchi (2010), mediante la utilización de modelos globales, apuntaron hacia un incremento del estrés hídrico en la región, fruto de la aridificación general de las zonas subtropicales y la expansión de las zonas subtropicales secas. Este hecho puede comprometer en gran medida las actividades económicas en el área de estudio, por lo que resulta necesario contrastar con registros climáticos de calidad si en las últimas décadas se asiste a un incremento de las condiciones de sequía y si el calentamiento observado puede estar aumentando su severidad, vía el aumento de la demanda de agua por parte de la atmósfera. Aunque actualmente existe un debate científico sobre los efectos del calentamiento en la severidad de las sequías a escala global (Sheffield et al., 2012; Dai, 2023; Trenberth et al., 2014), existen múltiples evidencias regionales que apuntan a que el calentamiento global está teniendo un notable impacto en la severidad de las sequías, observada tanto en ecosistemas naturales (Allen et al., 2015), cultivos (Lobell et al., 2013) y recursos hídricos (Vicente-Serrano et al., 2014).

Dada la importancia de las recurrentes sequías que impactan negativamente a la agricultura y a todas las actividades socio-económicas en la Cuenca Pacífico Norte, el principal objetivo de este estudio es analizar el comportamiento espacio-temporal de las sequías con dos indicadores a lo largo de cinco décadas. Todo ello, con la finalidad de identificar regiones que han presentado un comportamiento similar, y mejorar el conocimiento de cómo se comporta el fenómeno climático extremo más importante que afecta a esta región, que muestra un general aumento de las temperaturas a escala estacional y anual (Pavía et al., 2009). Para ello se han utilizado dos índices: el Índice de Precipitación Estandarizada (Standardized Precipitation Index, SPI) (McKee et al., 1993) y el Índice de Precipitación-Evapotranspiración Estandarizada (Standardized Precipitation Evapotranspiration Index, SPEI) (Vicente-Serrano et al., 2010a; VicenteSerrano, 2015a), para comprobar si los actuales procesos de calentamiento en el área de estudio están teniendo un cierto impacto en la severidad de las sequías durante el periodo analizado. Junto a ello, el estudio presenta una serie de objetivos secundarios: i) determinar la variabilidad espacio-temporal de la sequía a diferentes escalas temporales, ii) conocer la existencia de posibles tendencias en las sequías, y iii) determinar áreas homogéneas de acuerdo con el comportamiento de las sequías.

\section{2. Área de estudio}

La Cuenca Pacífico Norte comprende la totalidad del estado de Sinaloa y parte de los estados de Chihuahua, Durango, Zacatecas y Nayarit. Administrativamente está integrada por 51 municipios y una superficie de $152.013 \mathrm{~km}^{2}$. La población es de 4,42 millones de habitantes (CONAPO, 2014), albergando centros urbanos de gran población 
y elevada demanda de recursos hídricos como Culiacán (675.773), Durango (582.267) o Mazatlán (381.583). El clima dominante en la región es el templado subhúmedo, con zonas caracterizadas por un clima cálido subhúmedo. La temperatura media anual presenta fuertes variaciones espaciales, con un promedio de $20^{\circ} \mathrm{C}$ (CONAGUA, 2010). La precipitación media anual es de $747 \mathrm{~mm}$, aunque está afectada por una importante irregularidad y concentración estacional, ya que de julio a septiembre se recoge aproximadamente el $70 \%$ de la precipitación anual.

\section{Material y métodos}

\subsection{Base de datos}

Inicialmente se trabajó con las series mensuales de temperatura máxima $\left(\mathrm{T}_{\max }\right)$, temperatura mínima $\left(\mathrm{T}_{\min }\right)$ y precipitación $(\mathrm{P})$ disponibles en 803 estaciones meteorológicas, además de las series de evaporímetros Piché en 179 estaciones meteorológicas disponibles en el sistema de datos climatológicos CLImate COMputing project (CLICOM), administrado por el Servicio Meteorológico Nacional (SMN) (http://clicom-mex.cicese.mx). Muchas de las estaciones carecían de información de calidad, con excesivas lagunas de datos. Por ello, se seleccionaron aquellas series que al menos contuvieran 35 años con información de las tres variables $\left(\mathrm{T}_{\max } \mathrm{y}_{\min } \mathrm{y}\right.$ P), obteniendo 48 estaciones de las 803 iniciales, además de 22 series con registros de evaporímetros Piché, que suponen la disponibilidad de mediciones reales de demanda de agua por parte de la atmósfera en las garitas meteorológicas (SánchezLorenzo et al., 2014). Las series descartadas se utilizaron para el relleno de las series seleccionadas, mediante la aplicación de modelos de regresión simple, utilizando siempre la serie más correlacionada, con al menos diez años de datos comunes. La Tabla 1 muestra las estaciones seleccionadas, sus coordenadas y si cuentan con datos medidos de evaporación. La Figura 1 muestra la distribución espacial de las estaciones, que cubren la práctica totalidad de la Cuenca Pacífico Norte. Las series resultantes fueron sometidas a un cuidadoso control de calidad y homogeneización; el control de calidad es un paso fundamental para eliminar los datos incorrectos y comprobar la consistencia de los mismos (Feng et al., 2004). Dicho control estuvo basado en la comparación del rango de cada registro con el rango promedio de los datos registrados en estaciones adyacentes (Vicente-Serrano et al., 2010b). Para identificar y corregir las inhomogeneidades temporales, se utilizó el sistema HOMER (HOMogenizaton softwarE in R) http://www.homogenisation.org/ (Mestre et al., 2013). El método lleva a cabo una detección de inhomogeneidades a partir de las series pareadas y un análisis de varianza de dos factores para la corrección de los datos en el período de estudio de 1961-2010. Este método se identificó como uno de los que aportaban mejores resultados en la acción COST-HOME que testó diferentes métodos de homogeneidad (Venema et al., 2013). 
Tabla 1. Nombre, coordenadas y altitud de las estaciones utilizadas.

\begin{tabular}{|c|c|c|c|c|c|}
\hline Número de estacion & Estación & Longitud & Latitud & Altitud (m) & Evaporación \\
\hline 1 & Acaponeta, Acaponeta Dge & $-105,22$ & 22,3 & 31 & SI \\
\hline 2 & Ahome, Ahome & $-109,1$ & 25,55 & 80 & SI \\
\hline 3 & Boca-toma Sufragio (dge) & $-108,46$ & 26,49 & 152 & SI \\
\hline 4 & Canatlan, Canatlan & $-104,46$ & 24,31 & 2000 & SI \\
\hline 5 & Canelas, Canelas & $-106,34$ & 25,7 & 1360 & $\mathrm{NO}$ \\
\hline 6 & Culiacán, Culiacán & $-107,24$ & 24,49 & 40 & SI \\
\hline 7 & El Cantil,s. Papasquiaro & $-106,16$ & 24,57 & 2035 & $\mathrm{NO}$ \\
\hline 8 & El Carrizo, Ahome & $-109,19$ & 25,58 & 7 & $\mathrm{NO}$ \\
\hline 9 & El Fuerte, El Fuerte & $-108,37$ & 26,19 & 84 & $\mathrm{NO}$ \\
\hline 10 & el mahome, el fuerte & $-108,36$ & 26,31 & 12 & SI \\
\hline 11 & El Pino, Canatlan & $-104,54$ & 24,34 & 2004 & $\mathrm{NO}$ \\
\hline 12 & El Playon, Angostura & $-108,1$ & 25,15 & 5 & $\mathrm{NO}$ \\
\hline 13 & El saltito, nomb.de Dios & $-104,22$ & 24 & 1800 & $\mathrm{NO}$ \\
\hline 14 & El Tizate, Stgo. Ixcuintla & $-105,7$ & 21,45 & 19 & $\mathrm{NO}$ \\
\hline 15 & El Varejonal, Badiraguato & $-107,24$ & 25,6 & 122 & SI \\
\hline 16 & Francisco I.Madero (smn) & $-104,2$ & 24,24 & 1961 & $\mathrm{NO}$ \\
\hline 17 & Guachochic (cfe) & $-107,42$ & 26,49 & 1320 & $\mathrm{NO}$ \\
\hline 18 & Guadalupe Victoria (dge) & $-104,7$ & 24,26 & 1892 & NO \\
\hline 19 & Guadalupe y Calvo (smn) & $-106,58$ & 26,6 & 2279 & $\mathrm{NO}$ \\
\hline 20 & Guamuchil,(A.A.R.M.), S.A & $-108,49$ & 25,28 & 43 & SI \\
\hline 21 & Guatenipa, Badiraguato & $-107,13$ & 25,21 & 290 & $\mathrm{NO}$ \\
\hline 22 & Higuera de Zaragoza & $-109,19$ & 25,58 & 9 & SI \\
\hline 23 & Huites, Choix & $-108,22$ & 26,54 & 168 & SI \\
\hline 24 & Ixpalino, San Ignacio & $-106,37$ & 23,57 & 300 & SI \\
\hline 25 & Jaina, Sinaloa (dge) & $-108,1$ & 25,54 & 200 & SI \\
\hline 26 & Las Cañas, el Fuerte & $-108,36$ & 26,28 & 84 & SI \\
\hline 27 & Los Mochis, Ahome & -109 & 25,48 & 14 & SI \\
\hline 28 & Mocorito, Mocorito (dge) & $-107,55$ & 25,28 & 60 & $\mathrm{NO}$ \\
\hline 29 & Narciso Mendoza, Poanas & $-103,55$ & 24,1 & 1910 & SI \\
\hline 30 & Navios Viejos, Durango & $-105,3$ & 23,49 & 2492 & $\mathrm{NO}$ \\
\hline 31 & Ojito de Camellones & $-106,13$ & 25,4 & 2300 & $\mathrm{NO}$ \\
\hline 32 & Otinapa, Durango & $-105,1$ & 24,5 & 2400 & $\mathrm{NO}$ \\
\hline 33 & Peña del Aguila, Durango & $-105,19$ & 24,1 & 1896 & SI \\
\hline 34 & Pericos, Mocorito & $-107,46$ & 25,49 & 600 & SI \\
\hline 35 & Potrerillos, Concordia & $-105,52$ & 23,3 & 1470 & $\mathrm{NO}$ \\
\hline 36 & Rosamorada, Rosamorada & $-105,12$ & 22,9 & 44 & $\mathrm{NO}$ \\
\hline 37 & Rosario, Rosario & $-105,55$ & 23 & 27 & SI \\
\hline 38 & Ruiz Cortines, Guasave & $-108,43$ & 25,43 & 15 & $\mathrm{NO}$ \\
\hline 39 & San Blas, El Fuerte & $-108,46$ & 26,6 & 71 & SI \\
\hline 40 & San Jose de Acevedo & $-104,45$ & 23,46 & 1750 & $\mathrm{NO}$ \\
\hline 41 & San Pedro, Ruiz & $-105,9$ & 21,58 & 24 & $\mathrm{NO}$ \\
\hline 42 & Sanalona, Culiacan & $-107,9$ & 24,48 & 170 & SI \\
\hline 43 & Santa Cruz de Ayala, Cosala & $-108,54$ & 25,55 & 84 & SI \\
\hline 44 & Santiago Bayacora & $-104,37$ & 23,49 & 1900 & $\mathrm{NO}$ \\
\hline 45 & Siqueros, Mazatlan (dge) & $-106,15$ & 23,21 & 55 & $\mathrm{NO}$ \\
\hline 46 & Sombrerete, Sombrerete & $-103,38$ & 23,38 & 2379 & $\mathrm{NO}$ \\
\hline 47 & Vascogil, Canelas & $-106,24$ & 25,4 & 1220 & $\mathrm{NO}$ \\
\hline 48 & Vicente Guerrero & $-103,58$ & 23,43 & 1913 & SI \\
\hline
\end{tabular}




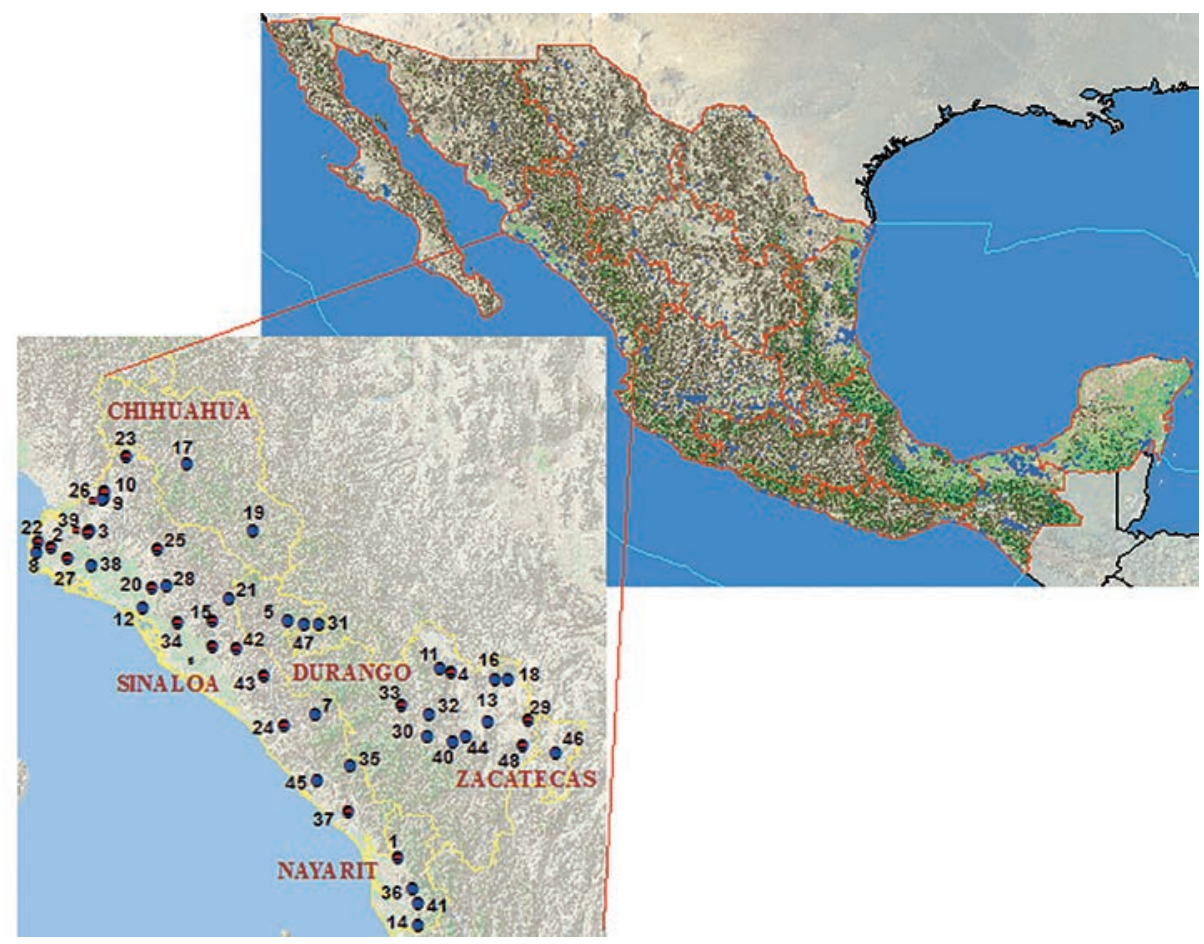

Figura 1. Localización del área de estudio. Estaciones meteorológicas con datos de Tmax, Tmin y P (círculos azules y rojos) y estaciones con Tmax, Tmin, P y Evaporación con datos de evaporímetros (círculos rojos).

\subsection{Generación de series regionales}

Además de las series individuales de cada una de las estaciones meteorológicas, se trabajó con una serie regional con la finalidad de recoger el patrón general del comportamiento de las sequías en el conjunto de la región. Para la obtención de la serie regional se utilizó el método desarrollado por Jones y Hulme (1996), a partir de la ponderación espacial de los registros mensuales en las diferentes estaciones disponibles. El factor de ponderación de cada estación fue la superficie representada por cada estación sobre el área total de la Cuenca Pacífico Norte, basada en el método de polígonos de Thiessen.

\subsection{Cálculo de los índices de sequía}

\subsubsection{El Índice de Precipitación Estandarizada (SPI)}

El Índice de Precipitación Estandarizada (SPI) es un índice que permite identificar condiciones de déficit y exceso de precipitación a diferentes escalas temporales. El índice se calcula mediante el ajuste de los datos mensuales de precipitación a una distribución de probabilidad (McKee et al., 1993). Para calcular el índice se precisa al menos 30 de 
años de datos mensuales, siendo preferible contar con al menos 50 años (Guttman, 1999), tal como se dispone en este trabajo (1961-2010). El SPI es un índice potente, flexible y sencillo de calcular y permite comparar áreas de pluviometría contrastada; de hecho, el único parámetro necesario para su cálculo es la precipitación. La principal crítica del SPI es que su cálculo se basa únicamente en datos de precipitación y no considera otras variables que influyen en la severidad de la sequía como puede ser la temperatura o, principalmente, la demanda de agua por parte de la atmósfera.

\subsubsection{El Índice de Precipitación Evapotranspiración Estandarizada (SPEI)}

El SPEI se calcula a partir de datos de precipitación y de demanda evaporativa por parte de la atmósfera, y tiene la ventaja de combinar el carácter multi-escalar del SPI con su capacidad para incluir los efectos de la demanda de agua por parte de la atmósfera en la severidad de la sequía. El procedimiento para el cálculo del índice se detalla en Vicente- Serrano et al. (2010a) y Beguería et al. (2014). Se trata de un índice que muestra igual sensibilidad a las variaciones de precipitación y evapotranspiración de referencia (ETo) (Vicente-Serrano et al., 2015) y que presenta ventajas respecto a otros indicadores para identificar los impactos de la sequía en diferentes sistemas naturales e hidrológicos (Vicente-Serrano et al., 2012)

La ETo se obtuvo mediante el método de Hargreaves y Samani (1985), basado en los datos de temperatura máxima, mínima y la radiación solar:

$$
\mathrm{ETo}=0.0023 \mathrm{RaR}^{0.5}(\mathrm{~T}+17.8)
$$

donde $\mathrm{R}$ es la diferencia entre la $\mathrm{T}_{\max } \mathrm{y} \mathrm{T}_{\text {min }}$ (temperaturas mensuales en ${ }^{\circ} \mathrm{C}$ ), $\mathrm{T}$ es la temperatura media mensual, $\mathrm{Ra}$ es la radiación solar expresada en E equivalente $\left(\mathrm{mm} \mathrm{dia}^{-1}\right)$ que depende de la latitud y día del año.

Este método es menos preciso que el recomendado por diferentes organismos, basado en el método de combinación de Penman-Monteith; sin embargo, dada la disponibilidad de datos en la zona de estudio, se trata de la aproximación más precisa que se pueda llevar a cabo. Diferentes estudios indican que las estimaciones de ETo basadas en la ecuación de Hargreaves muestran estimaciones muy similares a aquellas derivadas del método FAO-56 Penman-Monteith (Droogers y Allen, 2002).

Se calculó el SPI y el SPEI a las escalas temporales de “3”, "12”, “24” y “48” meses, en cada una de las estaciones meteorológicas y a partir de las series regionales de las diferentes variables. Del SPEI se calculó una versión diferente obtenida con datos de ETo, utilizando los datos de los evaporímetros como medida de la demanda de agua por parte de la atmósfera. Diferentes estudios señalan la paradójica evolución de la demanda de agua por parte de la atmósfera en diferentes regiones del mundo, donde bajo un escenario de calentamiento global se observan descensos en los valores de ETo (por ej., Roderick y Farquhar, 2002). Este hecho puede deberse al comportamiento observado por otras variables que controlan la demanda de agua por parte de la atmósfera, como la velocidad del viento, radiación o humedad relativa, y cuya evolución podría compensar la observada por la temperatura (McVicar et al., 2012). 


\subsection{Análisis de tendencias}

Los métodos más utilizados para el análisis de tendencias son las pruebas no paramétricas de correlación por rangos de Mann-Kendall y Spearman (Lanzante, 1996). Estas pruebas estadísticas identifican la presencia de cambios y describen si las tendencias son estadísticamente significativas. En este estudio se utilizó el test de Mann-Kendall para obtener las tendencias estacionales y anuales de los índices de sequía indicados más arriba. El nivel crítico seleccionado para aceptar un cambio como significativo fue de $\mathrm{p}<0.05$. El test indicado permite conocer si existen cambios significativos o no estadísticamente. Sin embargo, no permite determinar la magnitud de dichos cambios. Para ello se utilizaron modelos de regresión simple entre las series estacionales y anuales de los índices de sequía y las series de tiempo. La pendiente de la recta nos indica la magnitud de cambio por cada año. En este trabajo se multiplicó este resultado por los 50 años de datos disponibles, con la finalidad de saber cuánto ha cambiado la sequía, en unidades de media del índice, en el periodo de estudio.

\subsection{Regionalización del comportamiento de las sequías}

Finalmente, se llevó a cabo un análisis para comprobar las posibles diferencias espaciales en el comportamiento de las sequías dentro de la región Cuenca Pacífico Norte. Para ello se utilizó un Análisis de Componentes Principales (ACP) a partir de las series de los diferentes índices de sequía a la escala temporal de 12 meses. El ACP es el método más utilizado en los estudios de regionalización climática (P. Ej., Briffa et al., 1994; Huth, 2006). Este análisis permite retener los rangos comunes a todos los observatorios e identificar, a la vez, las particularidades de carácter más local, mediante un resumen de la información disponible. El primer componente representa la combinación lineal que absorbe el mayor porcentaje de varianza, el segundo el mayor porcentaje de la varianza no correlacionada con el primer componente y así sucesivamente. Se aplicó un PCA en modo S, donde los diferentes observatorios meteorológicos son las variables y los diferentes meses los casos (Serrano et al., 1999). Una vez obtenidos los componentes es recomendable su rotación, ya que la misma tiene el efecto de redistribuir la varianza y eliminar ambigüedades mientras se conserva la varianza entre el número de componentes no rotados. Se ha utilizado la rotación ortogonal Varimax (Kaiser, 1958), que es la opción más utilizada debido a su capacidad para obtener patrones más estables y físicamente explicables (Richman, 1986; White et al., 1991).

\section{Resultados}

\subsection{Evolución de las sequías}

En la Figura 2 se muestra un análisis de la evolución del SPI y el SPEI a partir de las series regionales obtenidas para el conjunto de la Cuenca Pacífico Norte en el periodo 1961-2010. Se muestra la evolución de los índices a escalas temporales de 3, 12, 24 y 48 meses. La serie regional de SPI muestra que los principales episodios de sequía se registraron en la década de 1980 y 2000. En 1982 es cuando se registra, en general, el episodio de mayor intensidad, aunque el episodio de 2003 presentó una duración y 
magnitud superiores. Las series de SPEI muestran un comportamiento temporal similar, reconociendo los mismos episodios de sequía que el SPI. Sin embargo, se observan notables diferencias entre la serie de SPEI obtenida mediante datos calculados de ETo y la serie calculada mediante observaciones de evaporímetros. Esta última muestra una mayor severidad de los episodios de sequía identificados hasta 1980 con relación a las series previas.
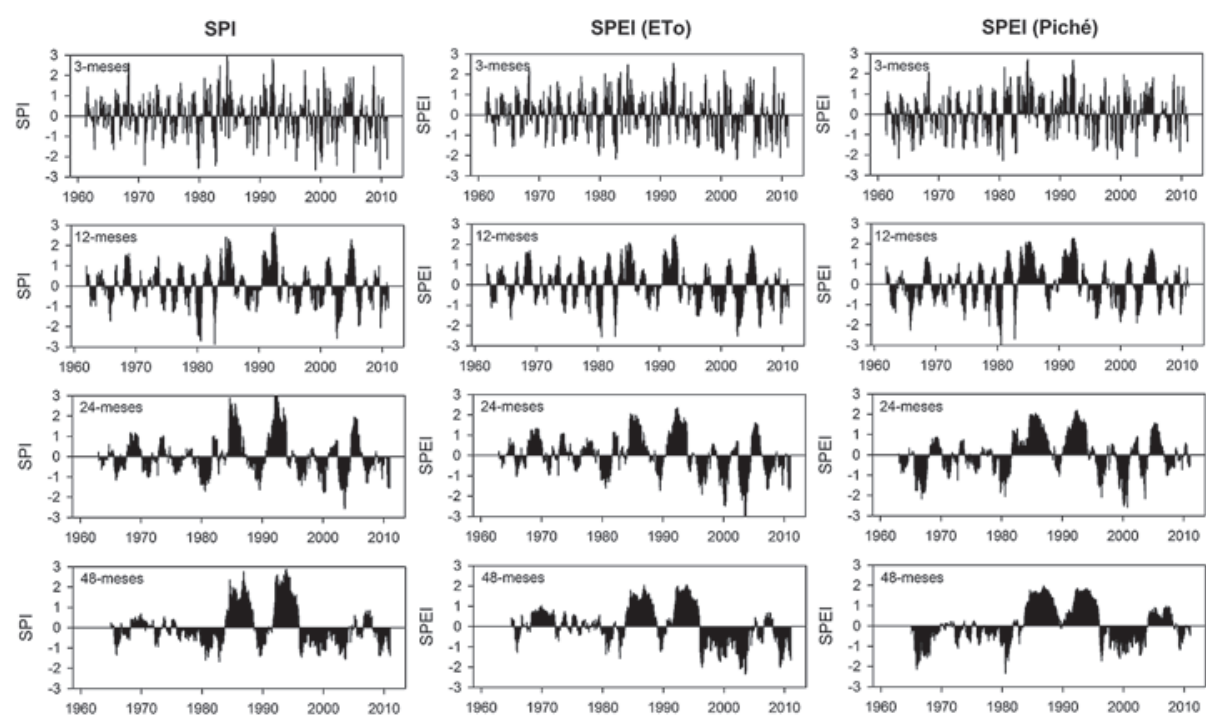

Figura 2. Serie regional con la evolución temporal (1961-2010) del SPI, SPEI (ETo) y SPEI (22 estaciones con datos de evaporímetros Piché) a las escalas temporales de 3, 6, 12 y 48 meses.

La Figura 3 muestra las diferencias entre la evolución de las series regionales de SPI y SPEI, y entre las series de SPEI obtenidas mediante ETo y aquellas obtenidas mediante tanques evaporímetros (para una adecuada comparación, dichas series regionales se han creado con los 22 observatorios comunes; los resultados utilizando la serie regional a partir de los 48 observatorios son similares -no mostrado-). La evolución muestra un incremento de las diferencias negativas entre SPEI basada en ETo y SPI, en las dos últimas décadas, que se refuerza considerablemente conforme aumenta la escala temporal del índice. Esto hace que durante los principales episodios de sequía observados en las últimas dos décadas (2003 y 2008-2009), la severidad de las sequías haya sido mayor utilizando el SPEI que el SPI. No obstante, este comportamiento también se observa calculando las diferencias entre el SPEI obtenido mediante datos de ETo y los datos de los evaporímetros. De hecho, la evolución hacia una mayor severidad de las sequías ha sido muy superior utilizando las series de ETo que las de los evaporímetros para calcular el SPEI. El ajuste de las series de evapotrímetros Piché y ETo es muy buena a escala regional (Figura 4), ya que aunque en los meses de mayor evaporación el método de Hargreaves sobreestima los valores 
observados en los evaporímetros Piché, las series de anomalías desestacionalizadas muestran una alta correlación $(\mathrm{r}=0,81)$. De hecho, las series de los evaporímetros Piché tienden a registrar valores anómalamente altos hasta 1975, con relación a las estimaciones de ETo (Fig. 5), lo que sugiere que o bien algunas variables que afectan en la variabilidad de la AED (por ej., velocidad del viento, humedad relativa o radiación), presentaron un comportamiento diferente al observado a partir de 1975, o bien pudieron existir problemas generalizados en la medición de esta variable. De hecho, el buen ajuste existente entre ETo y evaporación Piché desde $1980(\mathrm{r}=0,85)$ confiere una gran robustez a los cálculos de SPEI mediante datos de ETo obtenidos mediante la ecuación de Hargreaves, y hace sospechar que las series de evaporímetros pueden contener algunos problemas en las primeras décadas de medición. En los siguientes análisis únicamente se presentan los resultados de los índices obtenidos a partir de las series de ETo.
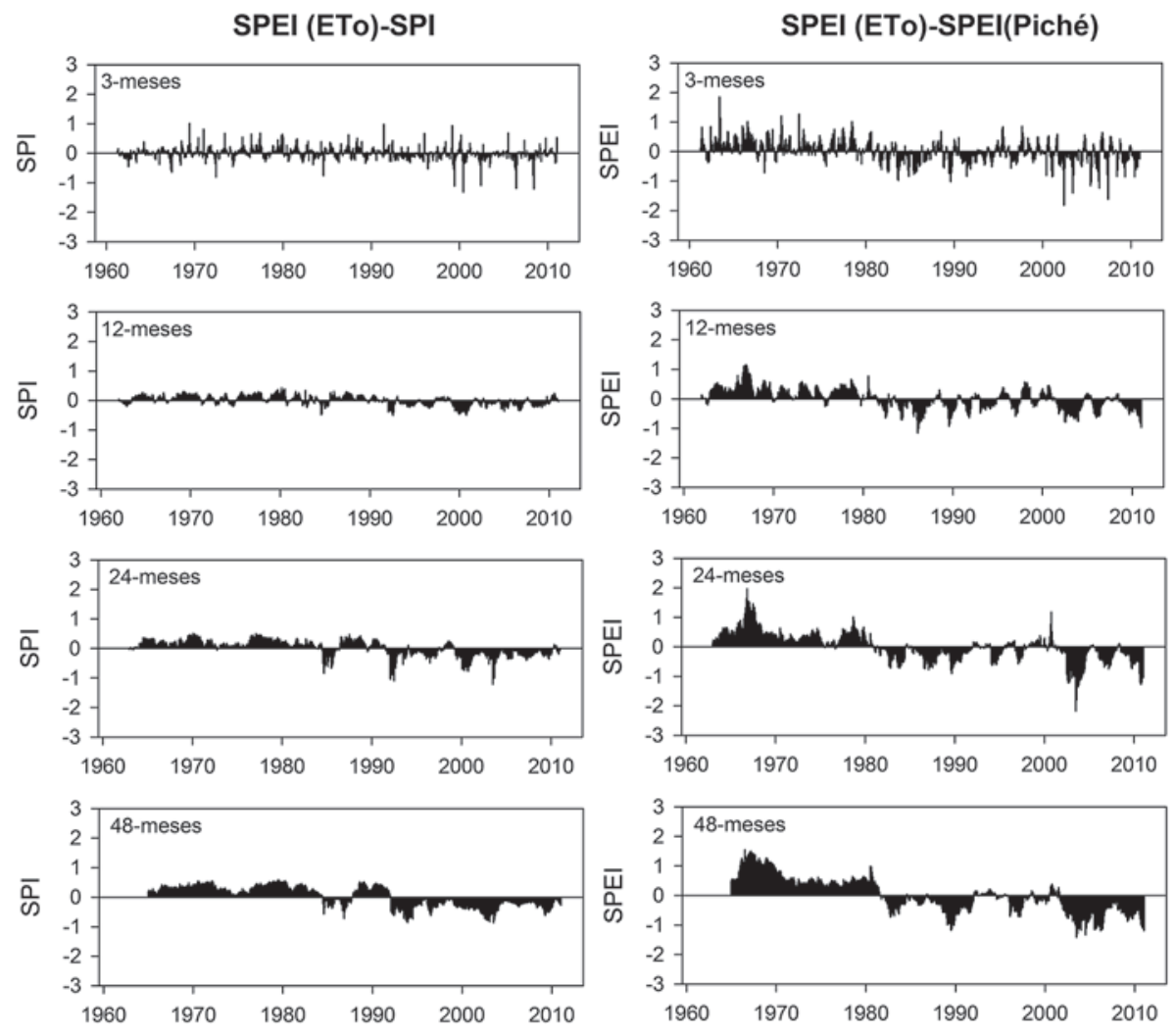

Figura 3. Diferencias entre SPEI y SPI (serie regional obtenida a partir de 48 estaciones), $y$ entre SPEI obtenido a partir de ETo y evaporímetros Piché (serie regional obtenida a partir de 22 estaciones) a las escalas temporales de 3, 12, 24 y 48 meses (1961-2010). 

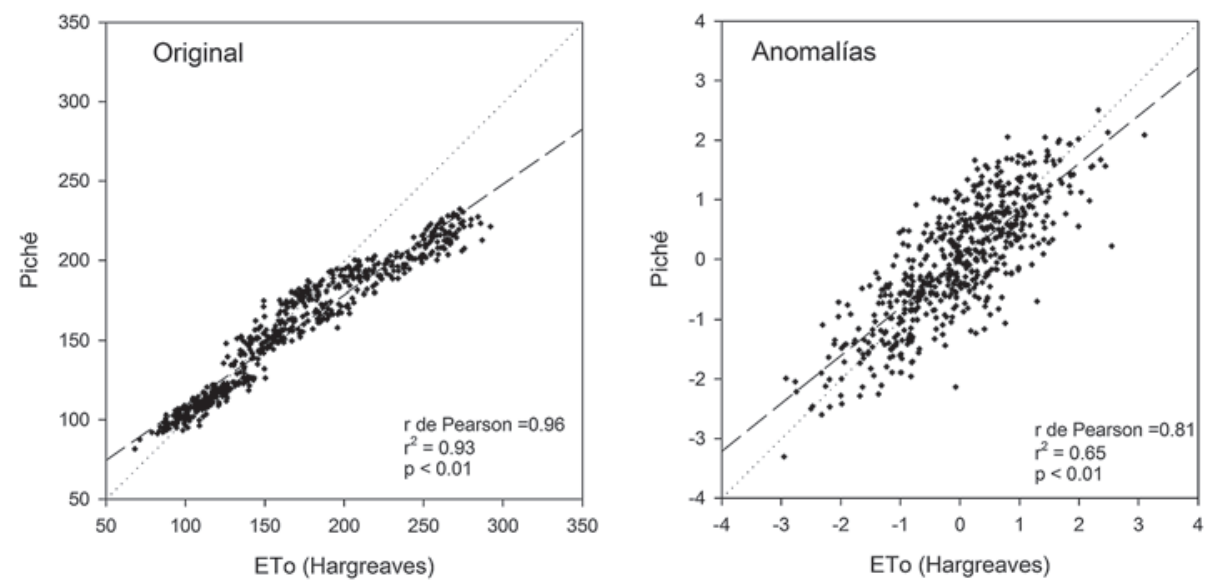

Figura 4. Relación entre los valores medios regionales observados de ETo y evaporación Piché y convertidos a anomalías desestacionalizadas.

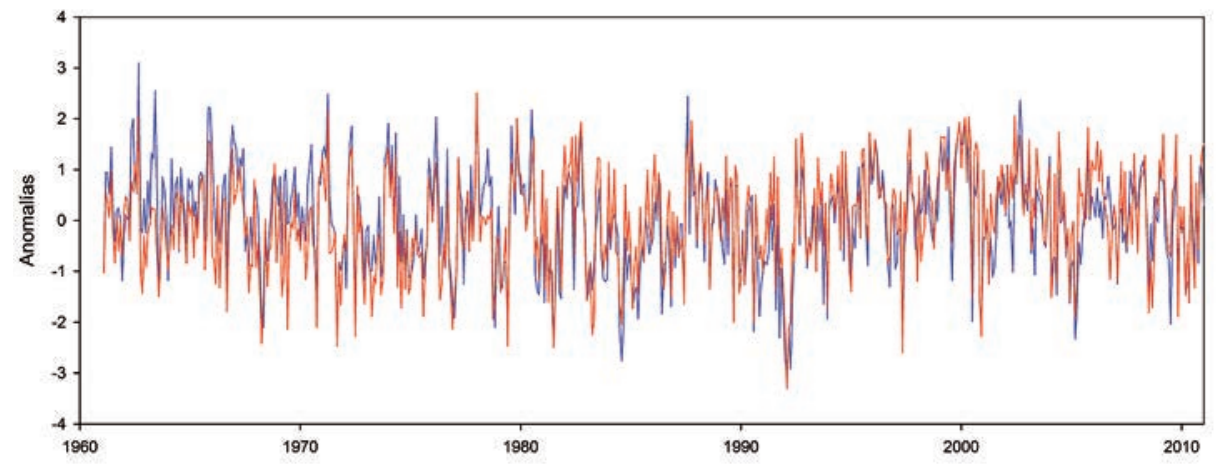

Figura 5. Evolución de las series de anomalías desestacionalizadas de evaporación Piché (azul) y ETo (rojo).

\subsection{Análisis de tendencias}

En la Figura 6 se muestran los resultados del análisis de tendencias en las series anuales (escala de 12 meses en diciembre) y estacionales (escala de 3 meses). A escala anual, tanto en el caso del SPI como del SPEI dominan los signos negativos, principalmente en el caso del SPEI, aunque son pocos los observatorios que muestran una tendencia estadísticamente significativa (4 en el caso de SPI y SPEI, respectivamente). No existen patrones espaciales generales en la tendencia de las sequías. 
A escala estacional se aprecian patrones más nítidos. En invierno se produce un dominante agravamiento de las condiciones de sequía en toda la región, particularmente en la zona central y oriental. En el caso del SPEI, todas las estaciones muestran una tendencia negativa durante el invierno. En primavera, aunque aparezcan observatorios con tendencias positivas, son las tendencias negativas las dominantes en el conjunto de la región. El 25\% de los observatorios muestran una tendencia negativa con el SPEI. En verano y otoño los patrones espaciales se hacen menos nítidos con ambos índices, aunque se comprueba que el SPEI refuerza las tendencias negativas en un buen número de estaciones meteorológicas.
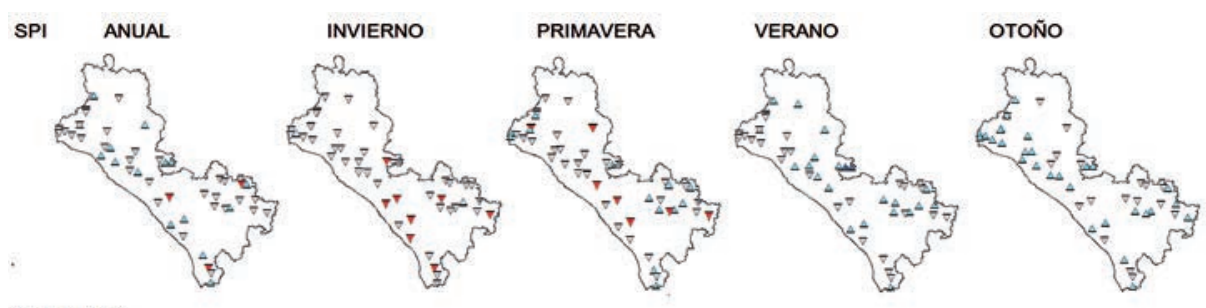

SPEI (ETo)
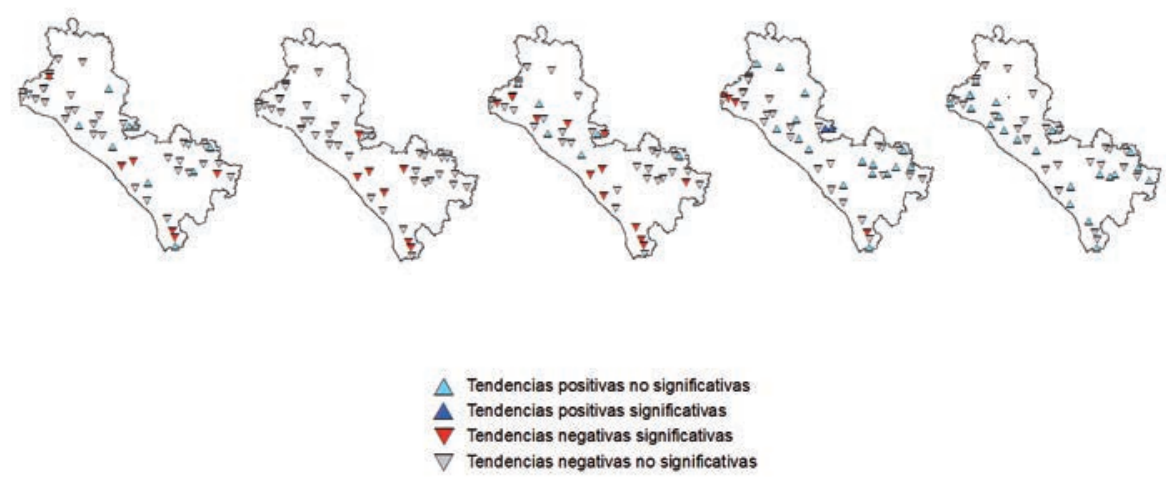

Figura 6. Tendencias estacionales y anuales en la evolución del SPI y SPEI (ETo)

(1961-2010).

La Figura 7 muestra la distribución espacial de los cambios en magnitud observados entre 1961 y 2010. Se muestran los valores interpolados espacialmente para facilitar la comparación. A escala anual (escala de 12 meses en diciembre) se observan diferencias importantes, con una clara tendencia hacia valores más negativos del SPEI de forma generalizada en toda el área de estudio. A nivel estacional, las diferencias entre los dos índices son más importantes en primavera y otoño. El SPEI muestra una tendencia a registrar cambios negativos de mayor magnitud que el SPI a escala anual y, sobre todo, en primavera, pero en el resto de estaciones del año el ajuste espacial entre los cambios observados con las series de SPEI y SPI resulta muy bueno (Fig. 8), indicando que los cambios experimentados por la ETo no han sido muy relevantes para producir un notable aumento de la severidad de las sequías durante el invierno, el verano y el otoño. 


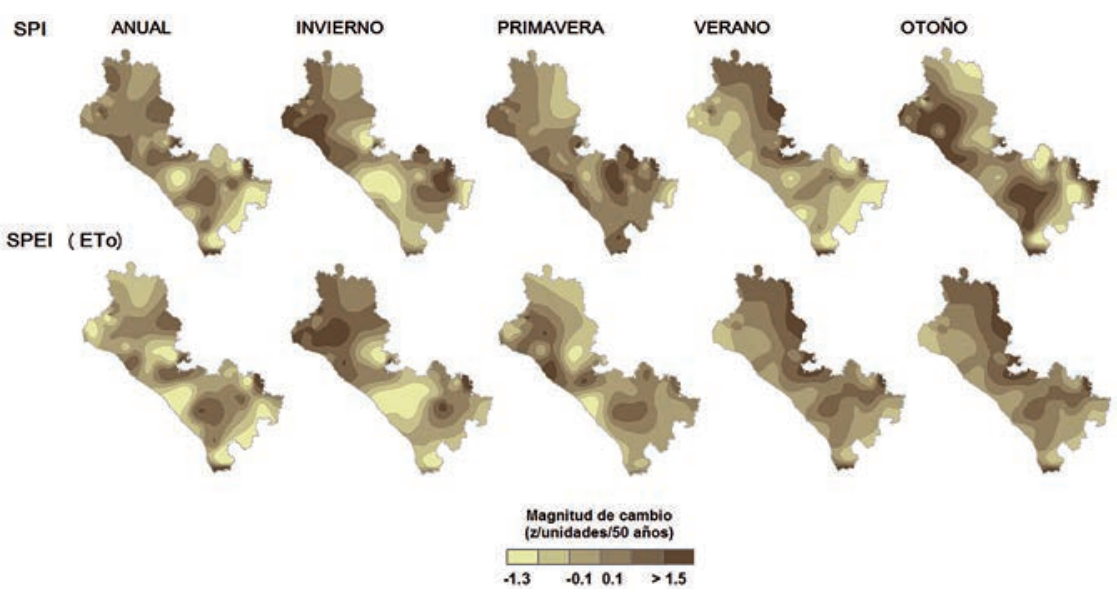

Figura 7. Magnitud de cambio anual y estacional en el SPI y SPEI (ETo) (1961-2010).
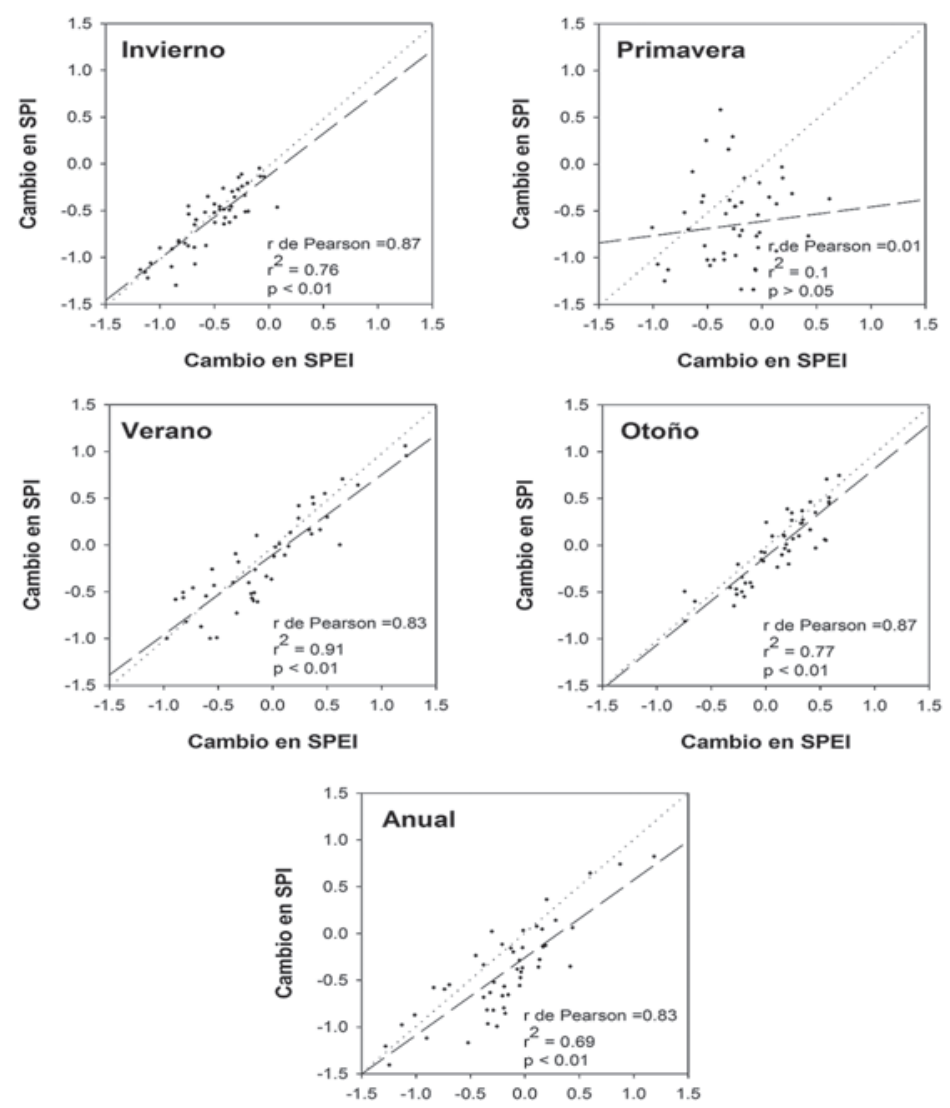

Figura 8. Relación entre los cambios estacionales y anuales observados en el SPI y SPEI en cada uno de los 48 observatorios meteorológicos. 


\subsection{Regionalización del comportamiento de las sequías}

Se ha comprobado que la evolución de las sequías presenta cierta variabilidad espacial en la región. La variabilidad es mayor considerando las series de SPEI que las de SPI, ya que se obtiene un mayor número de componentes principales (Fig. 9). No obstante, la mayor parte de regiones identificadas son coincidentes en el caso de los dos índices. El CP1 obtenido de las series de SPI representa gran parte del estado de Durango y una pequeña parte del estado de Zacatecas (33,2\% de la varianza explicada) (Fig. 10). La distribución de dicho componente se corresponde con el CP3 extraído de las series de SPEI (32,1\% de la varianza total). La evolución temporal de las series de ambos componentes es similar, aunque la intensidad de los episodios secos es algo superior en los últimos años considerando la serie del SPEI. El CP2 del SPI representa la parte septentrional de la Cuenca Pacífico Norte (27,7\%), con los principales episodios secos identificados en las décadas de 1970 y 2000. El patrón espacial corresponde al CP4 obtenido a partir del SPEI $(23,6 \%)$, aunque en este caso se observa una tendencia más clara hacia valores más bajos de SPEI en las últimas dos décadas analizadas. Finalmente, el CP3 del SPI representa la región noroccidental de la cuenca (13,9\%), que registra un episodio muy agudo en 1980 y otros menos extremos en la década de 2000. El CP2 de las series de SPEI (16,4\%) coincide espacialmente con el CP3 del SPI, aunque el episodio de 1980 fue de menor intensidad que el observado con la serie de SPI, mientras que los identificados en las décadas de 1990 y 2000 han presentado, en general, valores más negativos y una mayor duración.
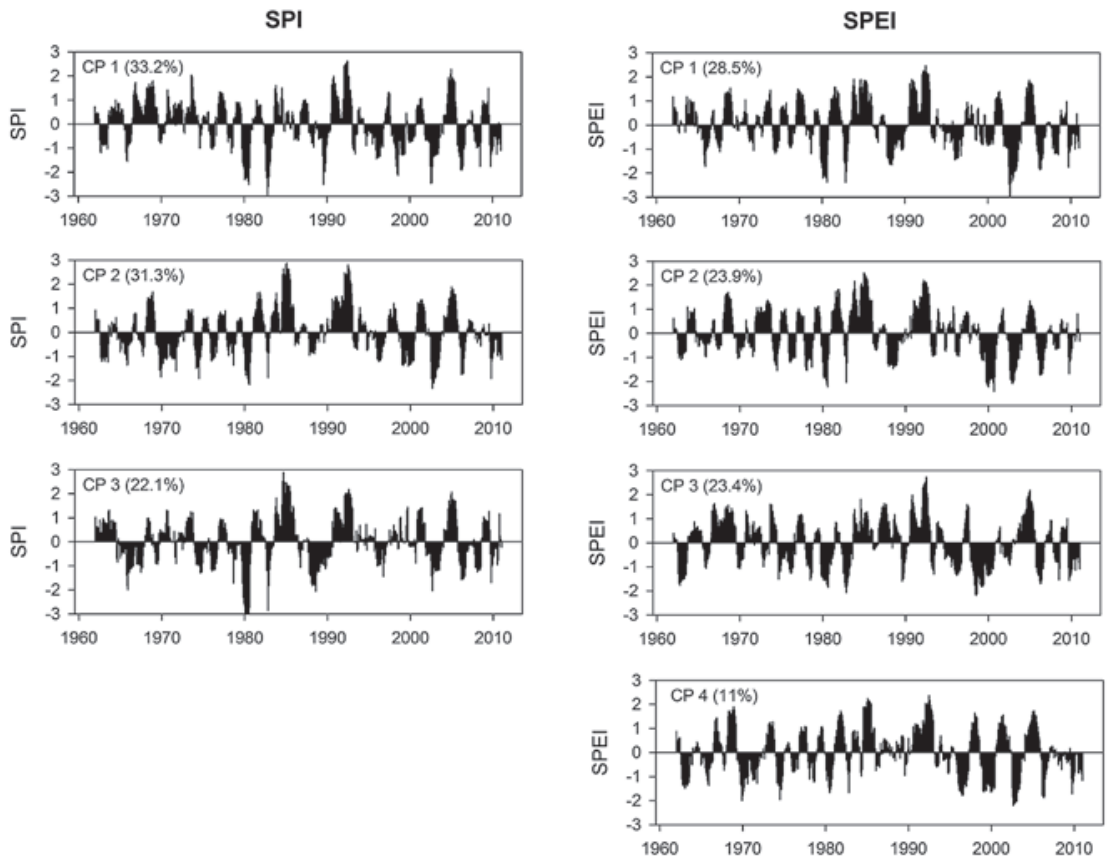

Figura 9. Evolución temporal de los componentes principales extraídos de las 48 series de SPI y SPEI a la escala temporal de 12 meses. Entre paréntesis se muestra el porcentaje de varianza explicado por cada componente. 


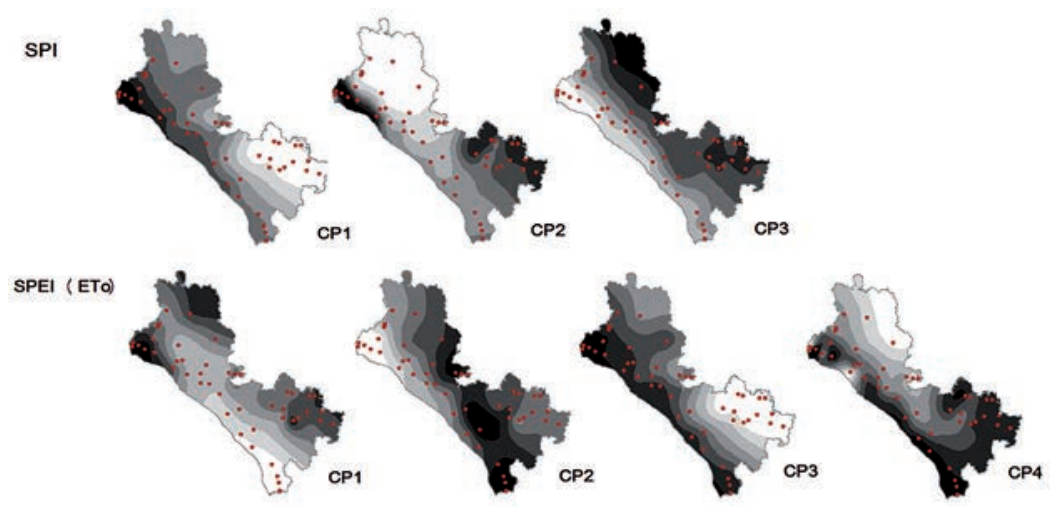

Figura 10. Distribución espacial de las cargas factoriales obtenidas a partir del análisis de las series de SPI y SPEI correspondientes a una escala temporal de 12 meses.

\section{Discusión y conclusiones}

Este trabajo ha analizado la variabilidad espacio-temporal de las sequías en la Cuenca Pacífico Norte de México entre 1961 y 2010. Se trata de la primera evaluación detallada de la variabilidad de las sequías en esta Cuenca, a partir de dos índices climáticos: El Índice de Precipitación Estandarizada (SPI) y el Índice de Precipitación Evapotranspiración Estandarizada (SPEI). Para ello se ha trabajado con un conjunto de estaciones meteorológicas en las que se testó su calidad y homogeneidad temporal. Se ha comprobado que las principales sequías afectaron a la región en las décadas de 1980 y 2000. Esta evolución coincide con la observada en otras regiones del norte de México, e incluso a nivel nacional, por parte de otros autores (Méndez et al., 2010; Bhattacharya et al., 2014).

Sin embargo, en este estudio hemos comprobado que existen algunas diferencias en función de los índices de sequía utilizados. Se ha comprobado que el SPEI presenta una tendencia dominante hacia unas condiciones de mayor sequía entre 1961 y 2010 en comparación a lo observado exclusivamente mediante las precipitaciones. Este patrón ha sido observado en otras regiones del mundo como la Península Ibérica (Vicente-Serrano et al., 2014), China (Yu et al., 2014; Wang et al., 2014); Korea (Nam et al., 2015), centroEuropa (Potop et al., 2014; Brázdil et al., 2015) o Bolivia (Vicente-Serrano et al., 2015b), entre otras. Este comportamiento se debe a la inclusión de la demanda de agua por parte de la atmósfera en el cálculo del SPEI, la cual se ha incrementado de forma dominante en las últimas tres décadas a consecuencia del incremento de la temperatura (Dai, 2013; Azorin-Molina et al., 2015).

Sin embargo, el proceso de evolución de las sequías no está exento de incertidumbres, pues diferentes autores han sugerido que a escala global no está claro que se esté produciendo un aumento de la demanda de agua por parte de la atmósfera (Sheffield et al., 2012), ya que el proceso no solamente depende de la temperatura, sino de otros fac- 
tores que determinan los componentes aerodinámico y radiativo (McVicar et al., 2012). En este estudio hemos utilizado series de evaporímetros Piché disponibles en la Cuenca del Pacífico Norte de México para comprobar si la utilización de observaciones de evaporación pueden complementar el cálculo de los índices de sequía. En general, se ha comprobado que el SPEI calculado mediante los datos desde evaporímetros Piché muestra un descenso inferior al observado con estimaciones de evapotranspiración de referencia (ETo) mediante el método de Hargreaves. No obstante, las divergencias se deben principalmente al comportamiento observado por la evaporación Piché durante los primeros 15 años disponibles, pues desde 1975 a 2010 existe un muy buen ajuste en las anomalías de la evaporación observada y la ETo. La razón de dicha divergencia en los primeros años puede deberse al comportamiento de otras variables que intervienen en la demanda de agua por parte de la atmósfera, como es el caso de la velocidad del viento, la humedad relativa o la radiación, o bien a problemas en la medición durante dicho periodo. Actualmente, es imposible aventurar la causa de dicho fenómeno, ya que no se dispone de series de dichas variables y que podrían matizar, por ejemplo, las notables diferencias espaciales en el comportamiento temporal de las sequías en primavera entre el SPI y el SPEI. No obstante, otros estudios ya han apuntado a la posible divergencia existente entre las tendencias temporales en las estimaciones y observaciones de la demanda de agua por parte de la atmósfera (Johnson y Sharma, 2007), como consecuencia de la perturbación introducida por los procesos de calentamiento global (Azorin-Molina et al., 2015).

En cualquier caso, se ha comprobado que la sequía es un fenómeno frecuente en la Cuenca del Pacífico Norte y que independientemente del impacto del posible estrés relacionado con la evapotranspiración, se registran numerosos episodios, algunos de ellos de gran intensidad, asociados a anomalías de precipitación durante largos periodos. Diferentes autores han señalado que los episodios más severos de sequía en el norte de México se ven favorecidos por la ocurrencia de fenómenos de la Niña (Méndez y Magaña, 2010). En este sentido los modelos climáticos predicen un agravamiento de los episodios de sequía en la región asociados a este tipo de fenómenos, que se acentuarían en un escenario de calentamiento global (Seager et al., 2009).

Los resultados de este estudio destacan que la sequía es un fenómeno complejo en la Cuenca Pacífico Norte de México, con algunas diferencias espaciales y una notable variabilidad temporal, lo que dificulta su monitorización y mitigación de sus impactos. Dados los negativos impactos que dicho fenómeno produce en la Cuenca Pacífico Norte, los resultados de este estudio pueden contribuir a una mejor valoración de los riesgos asociados, y a facilitar una mejor comprensión del fenómeno que permita determinar las posibles consecuencias futuras en un escenario de cambio climático.

\section{Agradecimientos}

Este trabajo ha recibido el soporte de los proyectos CGL2014-52135-C03-01 y Red de variabilidad y cambio climático RECLIM (CGL2014-517221-REDT) financiados por el Ministerio de Economía y Competitividad de España y FEDER. 


\section{Referencias bibliográficas}

Allen, C.D., Breshears, D.D., McDowell, N.G. 2015. On underestimation of global vulnerability to tree mortality and forest die-off from hotter drought in the Anthropocene. Ecosphere 6, 129.

Azorin-Molina, C., Vicente-Serrano, S.M., Sánchez-Lorenzo, A., McVicar, T.R., Morán-Tejeda, E., Revuelto, J., El Kenawy, A., Martín-Hernández, N., Tomás-Bruguera, M. 2015. Atmospheric evaporative demand observations, estimates and driving factors in Spain (1961-2011). Journal of Hydrology 523, 262-277.

Beguería, S., Vicente-Serrano, S.M., Reig, F., Latorre, B. 2014. Standardized Precipitation Evapotranspiration Index (SPEI) revisited: parameter fitting, evapotranspiration models, kernel weighting, tools, datasets and drought monitoring. International Journal of Climatology 34, 3001-3023.

Bhattacharya, T., Chiang, J.C.H. 2014. Spatial variability and mechanisms underlying El Niñoinduced droughts in Mexico. Climate Dynamics 43, 3309-3326.

Brázdil, R., Trnka, M., Mikšovský, J., Řezníčková, L., Dobrovolný, P. 2015. Spring-summer droughts in the Czech Land in 1805-2012 and their forcings. International Journal of Climatology 35, 1405-1421.

Briffa, K.R., Jones, P.D., Hulme, M. 1994. Summer moisture variability across Europe, 18921991: an analysis based on the Palmer drought severity index. International Journal of Climatology 14, 475-506.

CONAGUA 2010. Estadísticas del Agua en México, edición 2010. México. Disponible en: http:// www.conagua.gob.mx/CONAGUA07/Noticias/EAM2010.pdf.

CONAPO2014.Proyeccióndelapoblación2010-2050.Disponibleen:http://www.conapo.gob.mx/es/ CONAPO/Proyecciones.

Curtis, J.H., Hodell, D.A., Brenner, M. 1996. Climate variability on the Yucatan Peninsula (Mexico) during the past 3500 years, and implications for Maya cultural evolution. Quaternary Research 46, 37-47.

Dai, A., 2013. Increasing drought under global warming in observations and models. Nature Climate Change 3, 52-58.

Douriet, J.C., 2013. Pronóstico de Sequías en la Cuenca del río Culiacán, Sinaloa, México. En V Convención Cubana de Ciencias de la Tierra (Geociencias 2013), Palacio de Convenciones, La Habana.

Dracup, J.A., Lee, K., Paulson, E.G. 1980. On the definition of droughts. Water Resources Research 16, 297-302.

Droogers, P., Allen, R.G. 2002. Estimating reference evapotranspiration under inaccurate data conditions. Irrigation and Drainage Systems 16, 33-45.

Feng, S., Hu, Q., Qian, W. 2004. Quality control of daily meteorological data in China, 1951-2000: A new dataset. International Journal of Climatology 24, 853-870.

Guttman, N.B. 1999. Accepting the standardized precipitation index: A calculation algorithm. $J$. Amer. Water Resour. Assoc. 35, 311-322.

Hargreaves, G.L., Samani, Z.A. 1985. Reference crop evapotranspiration from temperature. Appl. Eng. Agric. 1, 96-99.

Hodell, D.A., Brenner, M., Curtis, J.H., Guilderson, T. 2001. Solar forcing of drought frequency in the Maya lowlands. Science 292, 1367-1370.

Huth, R. 2006. The effect of various methodological options on the detection of leading modes of sea level pressure variability. Tellus, Ser. A 58, 121-130.

Johnson, F., Sharma, A. 2007. Estimating evaporation - issues and challenges. En MODSIM07 - Land, Water and Environmental Management: Integrated Systems for Sustainability, Proceedings, pp. 589-595. 
Jones, P.D., Hulme, M. 1996. Calculating regional climatic time series for temperature and precipitation: methods and illustrations. International Journal of Climatology 16, 361-377.

Laliberte, A.S., Rango, A., Havstad, K., Paris, J.F., Becke, R.F., Amalia, L.G. 2004. Objectoriented image analysis for mapping shrub encroachment from 1937 to 2003 in southern New Mexico. Remote Sensing of Environment 93, 198-210.

Lanzante, J.R. 1996. Resistant, robust and non-parametric techniques for the analysis of climate data: theory and examples, including applications to historical radiosonde station data. International Journal of Climatology 16, 1197-1226.

Lloyd-Hughes, B. 2014. The impracticality of a universal drought definition. Theoretical and Applied Climatology 117, 607-611.

Lobell, D.B., Hammer, G.L., McLean, G., Messina, C., Roberts, M.J., Schlenker, W. 2013. The critical role of extreme heat for maize production in the United States. Nature Climate Change 3, 497-501.

Kaiser, H.E. 1958. The varimax criterion for analytic rotation in factor analysis. Psikometria 23, 187-200.

Magaña, V., Amador, J.A., Medina, S. 1999. The midsummer drought over Mexico and Central America. Journal of Climate 12, 1577-1588.

McKee, T.B.N., Doesken, J., Kleist, J., 1993. The relationship of drought frequency and duration to time scales. Eight Conf. on Applied Climatology. Anaheim, CA, Amer. Meteor. Soc. pp. 179-184.

McVicar, T.R., Roderick, M.L., Donohue, R.J., Van Niel, T.G. 2012. Less bluster ahead? Ecohydrological implications of global trends of terrestrial near-surface wind speeds. Ecohydrology 5, 381-388.

Méndez, M., Magaña, V. 2010. Regional aspects of prolonged meteorological droughts over Mexico and Central America. Journal of Climate 23, 1175-1188.

Mendoza, B., Velasco, V., Jáuregui, E. 2006. A study of historical droughts in southeastern Mexico. Journal of Climate 19, 2916-2934.

Mestre, O., Domokos, P., Picard, F., Aver, I., Robin, S., Lebarbier, E., Bohm, R., Aguilar, E., Guijarro, J., Vertachnik, G., Klancar, M., Dubuisson, B., Stepanek, P. 2013. HOMER: a homogenization software - methods and applications. Quart. Jour. of the Hungarian Meteorological Service 117, 47-67.

Mishra, A.K., Singh, V.P. 2010. A review of drought concepts. Journal of Hydrology 391, 202-216.

Mulholland, P.J., Best, G.R., Coutant, C.C., Hornberger, G.M., Meyer, J.L., Robinson, P.J. 1997. Effects of climate change on freshwater ecosystems of the south-eastern United States and the Gulf Coast of Mexico. Hydrological Processes 11, 949-970.

Nam, W.H., Hayes, M.J., Svoboda, M.D., Tadesse, T., Wilhite, D.A. 2015. Drought hazard assessment in the context of climate change for South Korea. Agricultural Water Management $160,106-117$.

Pavia, E.G., Graef, F., Reyes, J. 2009. Annual and seasonal surface air temperature trends in Mexico. International Journal of Climatology 29, 1324-1329.

Potop, V., Boroneanţ, C., Možný, M., Štěpánek, P., Skalák, P. 2014. Observed spatiotemporal characteristics of drought on various time scales over the Czech Republic. Theoretical and Applied Climatology 115, 563-581.

Ravelo, A.C., Sanz, R., Douriet, J.C. 2014. Detección, evaluación y pronóstico de las sequías en la región del Organismo de Cuenca Pacífico Norte, México. Agriscientia 31, 11-24.

Richman, M.B. 1986. Rotation of Principal Components. Journal of Climatology 6, 29-35.

Roderick, M.L., Farquhar, G.D. 2002. The cause of decreased pan evaporation over the past 50 years. Science 298, 1410-1411. 
Sánchez-Lorenzo, A., Vicente-Serrano, S.M., Wild, M., Calbó, J., Azorin-Molina, C., Peñuelas, J. 2014. Evaporation trends in Spain: a comparison of Class A pan and Piché atmometer measurements. Climate Research 61, 269-280.

Seager, R., Ting, M., Davis, M., Cane, M., Naik, N., Nakamura, J., Li, C., Cook, E., Stahle, D.W. 2009. Mexican drought: An observational modeling and tree ring study of variability and climate change. Atmosfera 22 1-31.

Seager, R., Vecchi, G.A. 2010. Greenhouse warming and the 21st century hydroclimate of southwestern North America. Proceedings of the National Academy of Sciences of the United States of America 107, 21277-21282.

Serrano, A., Garciacute, J., Mateos, V., Cancillo, M.L., Garrido, J. 1999. Monthly modes of variation of precipitation over the Iberian Peninsula. Journal of Climate 12, 2894-2919.

Sheffield, J., Wood, E.J., Roderick, M.L. 2012. Little change in global drought over the past 60 years. Nature 491, 435-438.

Trenberth, K.E., Guillemot, C.J. 1996. Physical processes involved in the 1988 drought and 1993 floods in North America. Journal of Climate 9, 1288-1298.

Trenberth, K.E., Dai, A., Van der Schrier, G., Jones, P.D., Barichivich, J., Briffa, K.R., Sheffield, J. 2014. Global warming and changes in drought. Nature Climate Change 4, 17-22.

Venema, V.K.C., Mestre, O., Aguilar, E., Auer, I., Guijarro, J.A., Domonkos, P., Vertacnik, G., Szentimrey, T., Stepanek, P., Zahradniek, P., Viarre, J., Müller-Westermeier, G., Lakatos, M., Williams, C.N., Menne, M.J., Lindau, R., Rasol, D., Rustemeier, E., Kolokythas, K., Marinova, T., Andresen, L., Acquaotta, F., Fratianni, S., Cheval, S., Klancar, M., Brunetti, M., Gruber, C., Prohom Duran, M., Likso, T., Esteban, P., Brandsma, T. 2012. Benchmarking homogenization algorithms for monthly data. Climate of the Past 8, 89-115.

Vicente-Serrano, S.M., Beguería, S., López-Moreno, J.I. 2010a. A multi-scalar drought index sensitive to global warming: the standardized precipitation evapotranspiration index - SPEI. Journal of Climate 23, 1696-1718.

Vicente-Serrano, S.M., Beguería, S., López-Moreno, J.I., García-Vera, M.A., Stepanek, P. 2010b. A complete daily precipitation database for North-East Spain: reconstruction, quality control and homogeneity. International Journal of Climatology 30, 1146-1163.

Vicente-Serrano, S.M., Beguería, S., López-Moreno, J.I. 2011. Comment on "Characteristics and trends in various forms of the Palmer Drought Severity Index (PDSI) during 1900-2008” by A. Dai. Journal of Geophysical Research-Atmosphere 116, D19112. Doi: 10.1029/2011JD016410.

Vicente-Serrano, S.M., Beguería, S., Lorenzo-Lacruz, J. 2012. Performance of drought indices for ecological, agricultural and hydrological applications. Earth Interactions 16, 1-27.

Vicente-Serrano, S.M., López-Moreno, J.I., Beguería, S., Lorenzo-Lacruz, J., Sánchez-Lorenzo, A., García-Ruiz, J.M., Azorín-Molina, C., Morán-Tejeda, E., Revuelto, J., Trigo, R., Coleho, F., Espejo, F. 2014. Evidence of increasing drought severity caused by temperature rise in southern Europe. Environmental Research Letters 9, 044001. Doi: 10.1088/17489326/9/4/044001.

Vicente-Serrano, S.M., Van der Schrier, G., Beguería, S., Azorin-Molina, C., Lopez-Moreno, J.I. 2015a. Contribution of precipitation and reference evapotranspiration to drought indices under different climates. Journal of Hydrology 426, 42-54.

Vicente-Serrano, S.M., Chura, O., López-Moreno, J.I., Azorín-Molina, C., Sánchez-Lorenzo, A., Aguilar, E., Morán-Tejeda, E., Trujillo, F., Martínez, R., Nieto, J.J. 2015b. Spatio-temporal variability of droughts in Bolivia: 1955-2012. International Journal of Climatology 35, 3024-3040.

Wang, W., Zhu, Y., Xu, R., Liu, J. 2014. Drought severity change in China during 1961-2012 indicated by SPI and SPEI. Natural Hazards 75, 2437-2451. 
White, D., Richman, H., Yarnal, B. 1991. Climate regionalization and rotation of principal components. International Journal of Climatology 11, 1-25.

Wilhite, D.A., Glantz. M.H. 1985. Understanding the Drought Phenomenon: the role or definitions. Water International 10, 111-120.

Wilhite, D.A. 2000. Drougth as Natural Hazard: Concepts and Definitions. En Wilhite, D.A. (ed.), Drought: an Assessment. Volume I, Routledge, New York, pp. 3-18.

Yu, M., Li, Q., Hayes, M.J., Svoboda, M.D., Heim, R.R. 2014. Are droughts becoming more frequent or severe in China based on the standardized precipitation evapotranspiration index: 1951-2010? International Journal of Climatology 34, 545-558. 